\title{
Sparse Image Reconstruction for Contrast Enhanced Cardiac Ultrasound using Diverging Waves
}

\author{
Antonio Stanziola ${ }^{1,4}$, Matthieu Toulemonde ${ }^{1}$, Virginie Papadopoulou ${ }^{1}$, Richard Corbett $^{2}$, \\ Neill Duncan ${ }^{2}$, Enrico Grisan ${ }^{3,4}$, and Mengxing Tang ${ }^{1}$ \\ ${ }^{1}$ Ultrasound Laboratory for Imaging and Sensing, Imperial College London, London, U.K. \\ ${ }^{2}$ Hammersmith Hospital, Imperial College Healthcare NHS Trust, London, U.K. \\ ${ }^{3}$ Dept. of Biomedical Engineering, Kings College London, London, U.K. \\ ${ }^{4}$ Dept. of Information Engineering (DEI), University of Padova, 35131, Italy
}

\begin{abstract}
Assessing cardiac function with trans-thoracic ultrasound is a challenging task, mainly due to its fast motion and its anatomical position which only allows for a narrow intercostal imaging window. These factors often lead to the use of diverging waves, even when contrast agents are employed. While capable of achieving a very high frames rate, an acquisition with diverging waves from a narrow aperture suffers from serious image quality degradation. In this regard, it is often impossible to mitigate this problem using common processing methods, such as coherent compounding.

In this study, we cast the problem of reconstructing the contrast enhanced ultrasound images as regularised inverse problem, analogous to the compressed sensing one, where the sensing matrix is fundamentally described by the delay operator associated with the time of flight. The results show that this framework can improve the Signal to Noise Ratio (SNR) of the image by up to $5.85 \mathrm{~dB}$ compared to delay and sum (DAS) and is therefore a promising way to reconstruct contrast enhanced cardiac images. The experiments also highlight that the way noise is modelled has a significant impact on the final image quality.

Index Terms-cardiac, ultrasound, contrast, sparse, compressed sensing
\end{abstract}

\section{INTRODUCTION}

High frame rate ultrasound (HFR US) imaging is a tomographic technique that, in the last decade, is slowly affirming itself as an alternative to classical focused ultrasound. The main difference between the two methods relies on the lack of focusing in HFR US, which allows to insonify the entire field of view with a single emission, while needing just a few steered emissions to achieve the same image quality as standard focused methods [1], [2]. HFR US has also been successfully used to image contrast agents [3], highly echogenic gas filled microbubbles injected in the blood stream, as well as being applied in contrast-enhanced echocardiography [4].

Imaging the heart and cardiovascular system with HFR US has several advantages. For example, the relatively small number of emissions reduces the presence of motion artefacts of classical images, which often reply upon hundreds of transmissions to generate a single image [5]; also, the fast

This work has been supported by an Engineering and Physical Sciences Research Council (EPSRC) grant EP/M011933/1, grant EP/M010961/1 and University of Padova intramural research project BIRD160889/16. We gratefully acknowledge the support of NVIDIA Corporation with the donation of the Titan Xp GPU used for this research. frame rate allows new precise flow quantification methods, such as Ultrasound Image Velocimetry [6], to be leveraged for cardiac applications.

The reconstruction stage of the image formation process is still largely based on DAS and coherent compounding [2], even if Fourier beamforming techniques [7] are becoming increasingly popular. One of the main advantages of DAS is its simple and highly parallelizable algorithmic structure, which allows for parallel reconstruction of each different pixel and therefore is a promising candidate for real-time image reconstruction of HFR US. However, the unfocused nature of HFR US transmissions requires compounding of multiple transmissions which, coupled with the fast velocity of blood flow in the heart, generates artifacts and changes in contrast quantification [8], while at the same time heavily reduces the frame rate.

If the measurement system, in this case the ultrasound acquisition platform, can be described using a linear operator, then compressive imaging is becoming an increasingly popular choice for improving the quality of the reconstructed images for many biomedical applications. For B-Mode ultrasound, Besson et al. have proposed a beamforming framework based on compressed sensing theory [9] that directly applies the methods developed for generic image reconstruction, demonstrating the possibility of reconstructing high-quality ultrasound images with limited number of measurements or transmissions. The core idea of the compressed sensing framework is that the target image belongs to a subset of the space of all possible images, and that the use of a regularization term helps steer the optimization towards an image that belongs to such a subset.

In this paper, we study how the feasibility of such a framework to reconstruct contrast enhanced cardiac images.

\section{THEORY}

\section{A. Time delay model and DAS}

Reflection US imaging relies on the collection of the waves scattered by a medium after being acoustically illuminated by a source wave-field $\phi_{t}(x, t)$. In the case of non-contrast imaging, for transmitted waves of low amplitude that generate a small 
perturbation of the particles position, the wave-field $\phi(x, t)$ can be described by the wave equation

$$
\nabla^{2} \phi-m(x) \frac{\partial}{\partial t} \phi=\phi_{t}
$$

where $m(x)=1 / c^{2}(x)$. In this case, the quantity of interest that one tries to derive from some measurements of $\phi$ is the speed of sound $(\mathrm{SoS}) c$. In biomedical ultrasound, the SoS is often approximated by a constant value (usually $c_{0}=1540$ $\mathrm{m} / \mathrm{s}$ for soft tissues) perturbed by a small function

$$
m(x)=\frac{1}{c_{0}}+\varepsilon \hat{m}(x) .
$$

With such a model, the quantity of interest that we aim to reconstruct is $\hat{m}(x)$. By further splitting the wave-field in incident and scattered components, $\phi=\phi_{i}+\phi_{s}$, the wave equation can be rewritten in its integral form and under mild conditions this equation can be iteratively solved for the scattered field using the Born approximation [10]. If one stops at the first iteration, the solution reads as

$$
\phi_{s} \approx-\varepsilon \iint \mathrm{d} \hat{x} \mathrm{~d} \hat{t}\left[G(x, \hat{x}, t-\hat{t}) \phi_{i}^{\prime \prime}(\hat{x}, \hat{t})\right] \hat{m}(\hat{x}) .
$$

Note that, in medical ultrasound, the Green's function $G$ most often refers to the constant velocity case.

\section{B. Linear vs non-linear response}

So far, we have assumed that the linear wave equation model holds. However, this is violated in the presence of microbubbles. At frequencies and pressures available in clinical scanners, microbubbles are often described using the RayleighPlesset equation [11]. This model has non-linear terms that describe microbubble oscillations as the output of a non-linear resonator: this predicts that contrast agent responses contain harmonics of the transmitted signals, a prediction that has been verified and is often used as the basis for discriminating microbubble signal from tissue echoes. [12]-[15].

For this reason, our measurement operator assumes that the emitted pulse is a Dirac delta, thus modelling the pulse as part of the image to be reconstructed, which is the reason why US images exhibit speckle. Lastly, we need to consider the fact that the wave-field is sampled only at finite positions, corresponding to the transducer element locations. If each element is approximated as a point-like transducer whose temporal impulse response presents a flat temporal spectrum, then eq. (3) reduces to eq. (1) of [16] without element directionality: this shows that the standard way of thinking about HFR US acquisitions is basically an application of the first Born approximation. We name the associated measurement model the Time of Flight (ToF) operator and denote it by the letter A.

The problem of US image reconstruction can therefore be stated as finding the best $\hat{m}(x)$ which follows the model

$$
\phi_{s}=A \hat{m}+\nu,
$$

where $\phi_{s}$ are the recorded acoustic data and $\nu$ is a noise vector that models acquisition noise and modelling errors.

\section{Model inversion}

Besson and colleagues, in [16], show that the DAS algorithm is basically the adjoint associated with the ToF operator, thus DAS is approximately equal to $A^{\top}$ : we refer the reader to this work for a more detailed discussion and for further comments on Compressed Sensing (CS) in the context of ultrasound beamforming.

Here, what we are interested in is the class of inversion algorithms that aim to find the original vector $m$ by solving the following optimization problem

$$
\underset{\hat{m}}{\arg \min } L\left(\phi_{s}, \hat{m}\right)+\lambda R(\hat{m}),
$$

where $L$ is a data-fidelity term and $R$ is a non-negative regularization functional weighted by a positive real factor $\lambda$.

The previous formulation can be derived from a Bayesian point of view: the key idea is to define a prior probability distribution over $m$, namely $P(m)$, such that the probability of seeing the data $\phi_{s}$ given the reflectivity function $m$ becomes

$$
P\left(\hat{m} \mid \phi_{s}\right)=\frac{P\left(\phi_{s} \mid \hat{m}\right) P(\hat{m})}{P\left(\phi_{s}\right)} .
$$

Maximizing the log likelihood of this probability with respect to $m$ gives

$$
\hat{\hat{m}}=\underset{\hat{m}}{\arg \min }-\log P\left(\phi_{s} \mid \hat{m}\right)-\log P(\hat{m}) .
$$

Taking this point of view, it is readily seen that the regularization term $\lambda R(\hat{m})$ corresponds to the prior probability associated to a given tissue reflectivity function $\hat{m}$. If we assume that noise is Gaussian and homoscedastic, then the loglikelihood for the data fidelity term becomes the well-known mean squared error (MSE) loss function

$$
-\log P\left(\phi_{s} \mid \hat{m}\right) \propto\left\|A \hat{m}-\phi_{s}\right\|^{2} .
$$

This is a questionable assumption, as noise in this formulation is mainly given by model inaccuracies, reflectors outside the field of view and electronic noise. While it may be hard to predict the probability distribution of the modelling inaccuracies, it is clear that it is clear that errors in the avergae SoS, for instance, will create a bias in the reconstruction error which is not necessarily Gaussian. This is especially true for cardiac imaging, as the waves travel trough very inhomogeneous media with large variations of SoS, such as the cardiac muscle and chamber, the latter being full of microbubbles. The same argument goes for errors in element sensitivity, ultrasound absorption, etc. The presence of reflectors outside the field of view will also likely result in a heteroscedastic covariance matrix, as its contribution will be more prominent for the latest part of the received RF data. Lastly, electronic noise is heteroscedastic, since ultrasound imaging often employs Time Gain Compensation (TGC) which results in a variable noise power across the RF data [17].

Another possibility is to assume, for example, that noise follows a Laplacian distribution, which leads to:

$$
-\log P\left(\phi_{s} \mid \hat{m}\right) \propto\left\|A \hat{m}-\phi_{s}\right\|_{1}
$$




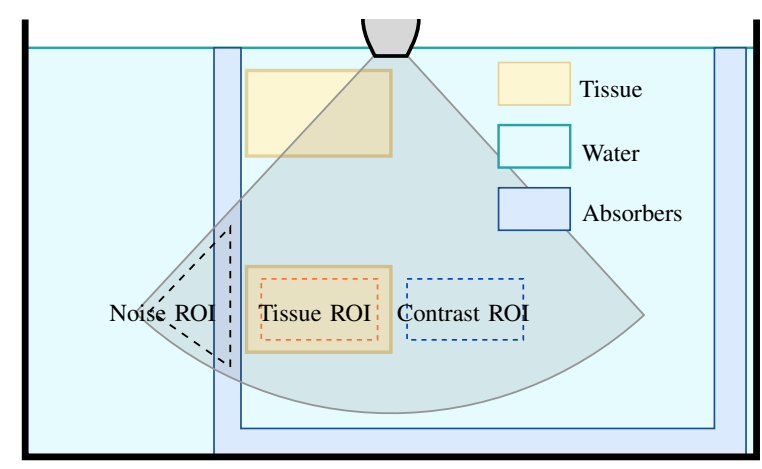

Fig. 1: In-vitro experimental setup.

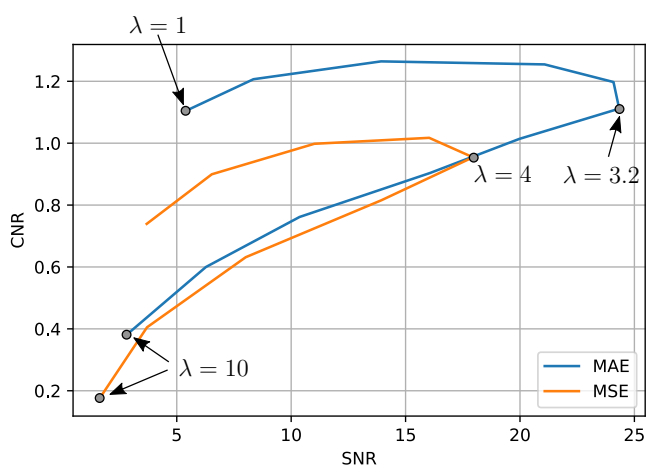

Fig. 2: SNR and CNR evolution for different regularization parameters.

The mean absolute error (MAE) will give less relative importance to large error values compared to MSE.

A second crucial question is how to choose a good prior $P(m)$. We postpone this point to further studies and for the time being we follow the work of [16] and use the same prior.

\section{METHODS}

\section{A. Ultrasound acquisitions}

Data is acquired using a P4-1 probe emitting a diverging wave of 3 cycles and center frequency of $1.5 \mathrm{MHz}$. Pulse inversion is used to highlight contrast signals and the received RF data are high-pass filtered, using a $5^{\text {th }}$ order Butterworth filtered with cut-off frequency at $2.7 \mathrm{MHz}$.

The phantom, depicted in Fig. 1, consists of two pieces of tissue mimicking material immersed in a tank of degassed water with Sonovue microbubbles dispersed at clinical concentration. The images are reconstructed using the full received data or with half of channels missing, to test the power of the regularized formulation with missing data.

\section{B. Sparse problem inversion}

The reconstruction of the image is done by solving

$$
\hat{m}=\underset{\hat{m}}{\arg \min }\left\|A \hat{m}-\phi_{s}\right\|_{p}^{2}+\lambda\|\Psi \hat{m}\|_{1},
$$

where $\Psi$ is defined as in eq. (21) of [16] and $p \in\{1,2\}$. The optimization is done using sub-gradient descent in PyTorch

\begin{tabular}{rcccc} 
& Contrast & CTNR & SNR & CNR \\
\hline DAS & -0.81 & -7.96 & 7.66 & -0.27 \\
MAE & 3.85 & -1.55 & $\mathbf{1 3 . 5 1}$ & $\mathbf{1 . 0 0}$ \\
MSE & $\mathbf{6 . 1 5}$ & $\mathbf{- 0 . 9 1}$ & 9.21 & -0.27 \\
\hline DAS (half channels) & 1.14 & -5.09 & 3.20 & -0.81 \\
MAE (half channels) & 2.60 & -4.01 & $\mathbf{1 0 . 2 8}$ & -0.56 \\
MSE (half channels) & $\mathbf{2 . 8 6}$ & $\mathbf{- 3 . 4 7}$ & 8.18 & $\mathbf{- 0 . 4 1}$
\end{tabular}

TABLE I: Evaluation of the various reconstruction methods $(\mathrm{dB})$.

[18] with momentum and a step size of 0.01 . The operator $A$ includes element directionality at the second harmonic [19] as well as the spherical amplitude decay. The use of $p=1$ and $p=2$ is denoted as MAE and MSE respectively throughout the manuscript.

\section{Analysis}

Image quality is measured by comparing the signal in the microbubble region to tissue and noise, whose ROIs are depicted in Fig. 1. The following metrics are used:

$$
\begin{aligned}
\mathrm{SNR} & =\frac{\mu_{\text {contrast }}^{2}}{\mu_{\text {noise }}^{2}} \quad \mathrm{CNR}=\frac{\left\|\mu_{\text {contrast }}-\mu_{\text {noise }}\right\|^{2}}{\sigma_{\text {contrast }}^{2}+\sigma_{\text {noise }}^{2}} \\
\text { Contrast } & =\frac{\mu_{\text {contrast }}^{2}}{\mu_{\text {tissue }}^{2}} \\
\mathrm{CTNR} & =\frac{\left\|\mu_{\text {contrast }}-\mu_{\text {tissue }}\right\|^{2}}{\sigma_{\text {contrast }}^{2}+\sigma_{\text {tissue }}^{2}},
\end{aligned}
$$

where $\mu$ and $\sigma^{2}$ denote the mean and variance of the signal in the ROI.

\section{RESULTS AND DISCUSSION}

The solution of the regularized problem strongly depends on the choice of the parameter $\lambda$. To find a value that gives a good balance between noise suppression and discarded data, we plot the value of SNR and CNR for different $\lambda$ in Fig. 2. The two problems give very different solutions in terms of SNR and CNR, with the MAE formulation capable of achieving SNR values of almost 25 and CNR values of more than 1.2, while the MSE formulation can reach at most 17.5 and 1.0 respectively. This already highlights the importance of carefully evaluating the noise model for the problem at hand.

The images reconstructed using the best regularization weight are shown in Fig. 3 and their quality is analyzed in Tab. I. The results show that MAE always outperforms MSE in terms of SNR, by $4.3 \mathrm{~dB}$ and $2.1 \mathrm{~dB}$ for the full and half channels reconstructions respectively. Both methods outperform DAS in all the image quality metrics, showing that such regularized framework is suitable also for reconstructing contrast enhanced cardiac images. At the same time, the values of CNR for MAE are slightly better $(+0.73 \mathrm{~dB})$ or comparable $(-0.15 \mathrm{~dB})$ to the MSE case. The values of Contrast and CTNR are always higher for the MSE case: while this may seem to suggest that the MSE method is better at suppressing tissue signals, another possibility is that the MSE method tends to slightly suppress weak signals and, being that the tissue signal is weaker than the contrast one, it shows improved Contrast 


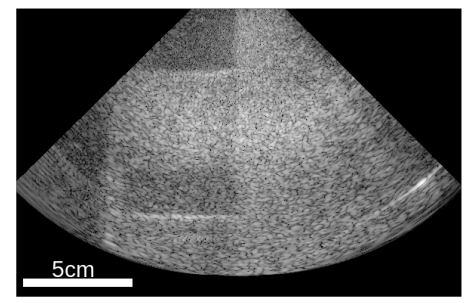

(a) DAS

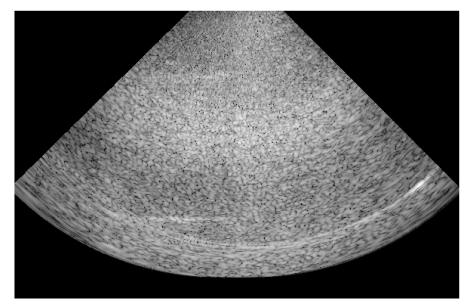

(d) DAS (half channels)

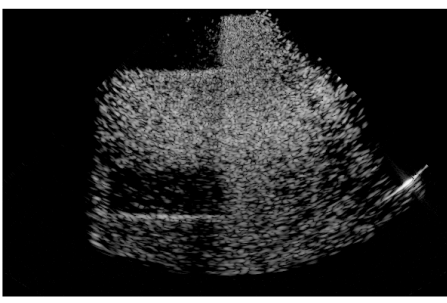

(b) $\operatorname{MSE}(\lambda=4)$

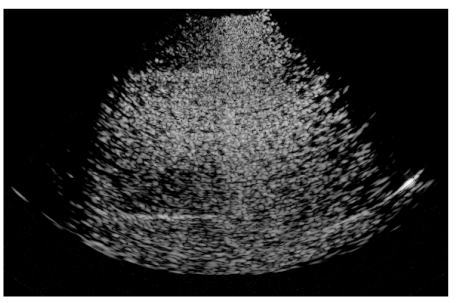

(e) $\operatorname{MSE}(\lambda=4$, half channels $)$

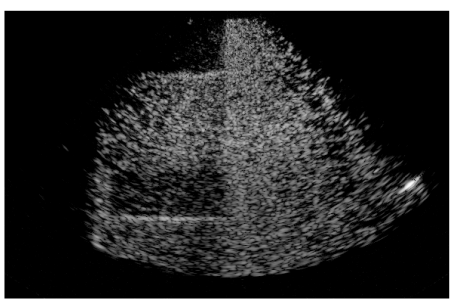

(c) $\operatorname{MSE}(\lambda=3)$

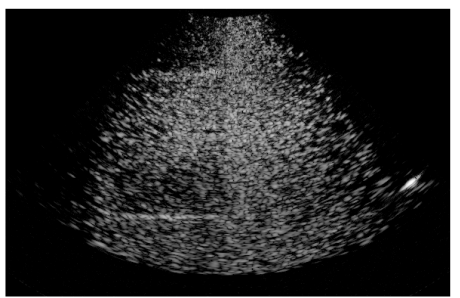

(f) $\operatorname{MAE}(\lambda=3$, half channels)

Fig. 3: PI images reconstructed using the various methods for a full aperture (top) and when half of the channels are missing (bottom). Images are shown with a dynamic range of $30 \mathrm{~dB}$, normalized by their own maximum

and CTR measures with respect to MAE. In particular, this possibility can be detrimental for applications such as the evaluation of the myocardial circulation, as it may suppress the weak contrast signal expressed in the cardiac muscle.

\section{Conclusions}

This works shows that sparsity regularized inversion of contrast acquisitions with time of flight model improves the image quality compared with DAS and can be used to enhance the reconstruction of contrast enhanced cardiac acquisitions. However, it also suggests that the exact formulations of the problem, especially in terms of noise, has a significant impact on the final image quality and must be carefully evaluated.

\section{REFERENCES}

[1] G. Montaldo, M. Tanter, J. Bercoff, N. Benech, and M. Fink, "Coherent plane-wave compounding for very high frame rate ultrasonography and transient elastography," Ultrasonics, Ferroelectrics, and Frequency Control, IEEE Transactions on, vol. 56, no. 3, pp. 489-506, 2009.

[2] M. Cikes, L. Tong, G. R. Sutherland, and J. D'Hooge, "Ultrafast cardiac ultrasound imaging: Technical principles, applications, and clinical benefits," JACC: Cardiovascular Imaging, vol. 7, no. 8, pp. 812-823, 2014.

[3] D. Cosgrove, "Ultrasound contrast agents: An overview," European Journal of Radiology, vol. 60, no. 3, pp. 324-330, 2006.

[4] M. Toulemonde, Y. Li, S. Lin, F. Cordonnier, M. Butler, W. C. Duncan, R. J. Eckersley, V. Sboros, and M.-X. Tang, "High-Frame-Rate Contrast Echocardiography Using Diverging Waves: Initial In Vitro and In Vivo Evaluation," IEEE Transactions on Ultrasonics, Ferroelectrics, and Frequency Control, vol. 65, pp. 2212-2221, Dec. 2018.

[5] T. L. Szabo, Diagnostic Ultrasound Imaging: Inside Out. 2013.

[6] C. H. Leow, E. Bazigou, R. J. Eckersley, A. C. H. Yu, P. D. Weinberg, and M.-X. Tang, "Flow Velocity Mapping Using Contrast Enhanced High-Frame-Rate Plane Wave Ultrasound and Image Tracking: Methods and Initial in Vitro and in Vivo Evaluation.," Ultrasound in medicine \& biology, vol. 41, no. 11, pp. 2913-2925, 2015.

[7] M. Zhang, A. Besson, R. E. Carrillo, M. Viallon, D. Garcia, J.-p. Thiran, D. Friboulet, and O. Bernard, "Extension of Fourier-based techniques for ultrafast imaging in ultrasound with diverging waves," pp. 1-12.
[8] M. Toulemonde, W. C. Duncan, A. Stanziola, and R. J. Eckersley, "Effects of motion on high frame rate contrast enhanced echocardiography and its correction," IEEE International Ultrasonics Symposium (IUS), pp. 31-34, 2017.

[9] A. Besson, R. E. Carrillo, O. Bernard, Y. Wiaux, and J.-P. Thiran, "Compressed delay-and-sum beamforming for ultrafast ultrasound imaging," 2016 IEEE International Conference on Image Processing (ICIP), pp. 2509-2513, 2016.

[10] L. Demanet, "Waves and Imaging Class notes - 18.325," 2016.

[11] T. Faez, M. Emmer, K. Kooiman, M. Versluis, A. F. W. van der Steen, and N. de Jong, "20 years of ultrasound contrast agent modeling," IEEE Transactions on Ultrasonics, Ferroelectrics and Frequency Control, vol. 60, Jan. 2013.

[12] A. Stanziola, M. Toulemonde, Y. Yildiz, R. Eckersley, and M.-X. Tang, "Ultrasound imaging with microbubbles," IEEE Signal Processing Magazine, vol. 33, no. 2, 2016.

[13] V. Mahue, J. M. Mari, R. J. Eckersley, and M. Tang, "Comparison of pulse subtraction doppler and pulse inversion doppler," IEEE Transactions on Ultrasonics, Ferroelectrics, and Frequency Control, vol. 58, pp. 73-81, Jan. 2011.

[14] F. Lin, C. Cachard, F. Varray, and O. Basset, "Generalization of Multipulse Transmission Techniques for Ultrasound Imaging," Ultrasonic Imaging, vol. 37, pp. 294-311, Oct. 2015.

[15] C. Tremblay-darveau, R. Williams, L. Milot, M. Bruce, and P. N. Burns, "Combined Perfusion and Doppler Imaging Using Plane-Wave Nonlinear Detection and Microbubble Contrast Agents," IEEE transactions on ultrasonics, ferroelectrics, and frequency control, vol. 61, no. 12, pp. 1988-2000, 2014.

[16] A. Besson, D. Perdios, F. Martinez, Z. Chen, R. E. Carrillo, M. Arditi, Y. Wiaux, and J.-P. Thiran, "Ultrafast Ultrasound Imaging as an Inverse Problem: Matrix-Free Sparse Image Reconstruction," IEEE Transactions on Ultrasonics, Ferroelectrics, and Frequency Control, vol. 65, pp. 339355, Mar. 2018.

[17] C. Huang, P. Song, P. Gong, J. D. Trzasko, A. Manduca, and S. Chen, "Debiasing-based Noise Suppression for Ultrafast Ultrasound Microvessel Imaging," IEEE Transactions on Ultrasonics, Ferroelectrics, and Frequency Control, pp. 1-1, 2019.

[18] A. Paszke, S. Gross, S. Chintala, G. Chanan, E. Yang, Z. DeVito, Z. Lin, A. Desmaison, L. Antiga, and A. Lerer, "Automatic differentiation in PyTorch,” p. 4, 2017.

[19] A. R. Selfridge, G. S. Kino, and B. T. KhuriYakub, "A theory for the radiation pattern of a narrowstrip acoustic transducer," Applied Physics Letters, vol. 37, pp. 35-36, July 1980. 